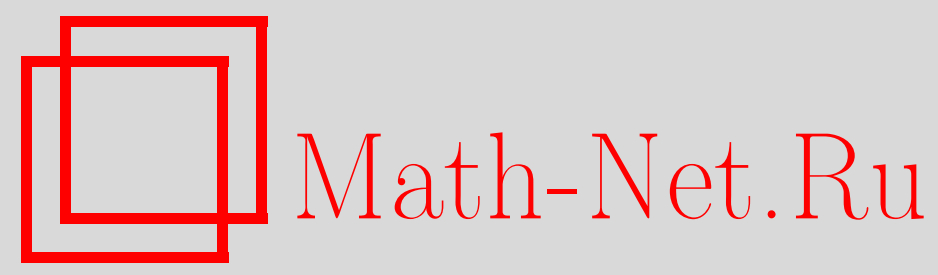

В. Ф. Гапошкин, О сходимости почти всюду средних Рисса однородных случайных полей, Теория вероятн. и ее примен., 1997, том 42, выпуск 3, 461-472

DOI: https://doi.org/10.4213/tvp1944

Использование Общероссийского математического портала Math-Net.Ru подразумевает, что вы прочитали и согласны с пользовательским соглашением http://www . mathnet.ru/rus/agreement

Параметры загрузки:

IP : 54.157 .27 .8

26 апреля 2023 г., 12:00:50

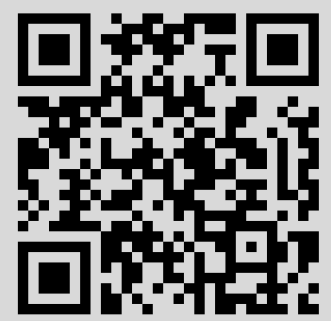


(C) 1997 г.

ГАПГОІККИН В. Ф.*

\section{О СХОДИМОСТИ ПОЧТИ ВСЮДУ СРЕДНИХ РИССА ОДНОРОДНЫХ СЛУЧАЙНЫХ ПОЛЕЙ ${ }^{1)}$}

Изучаются весовые средние однородных (в широком смысле) случайных полей в $\mathbf{R}^{k}$ со сферически симметричными весами. Получено представление этих средних, позволяющее доказать критерий суммирования почти всюду однородных случайных полей, а также ряд следствий.

Ключевые слова и фразы: однородные в широком смысле случайные поля, спектральная мера поля, весовые средние, корреляционная функция, суммируемость почти всюду, логарифмические средние Рисса.

Пусть $\left(X(t), t \in \mathbf{R}^{k}\right)$ - однородное случайное поле второго порядка, $\mathbf{E} X(t)=0, \quad \mathbf{E}|X(t)|^{2}=\sigma^{2}, \quad \overline{\mathbf{E} X(t)} X(t+u)=K(u), \quad u, t \in \mathbf{R}^{k}$, $Z(d \lambda)$ - спектральная мера поля, имеюшая ортогональные прирашения, и спектральное представление поля имеет вид

$$
X(t)=\int_{\mathbf{R}^{k}} \exp \{i(t, \lambda)\} Z(d \lambda) .
$$

Пусть $b_{1}(u)$ - положительная весовая функция на $(0, \infty)$,

$$
\begin{aligned}
b(u) & =b_{1}(u) u^{k-1}, \\
B_{1}(r) & =\int_{|t| \leqslant r} b_{1}(|t|) d t=c_{k} \int_{0}^{\tau} u^{k-1} b_{1}(u) d u=c_{k} B(r), \\
c_{k} & =k V_{k}, \quad \text { где } \quad V_{k}=\pi^{k / 2} \Gamma^{-1}\left(\frac{k}{2}+1\right)
\end{aligned}
$$

есть объем единичного шара.

Предположим, что $\lim _{r \rightarrow \infty} B(r)=\infty$.

*Московский государственный университет путей сообшения (МИИТ), ул. Образщова, 15, 103055 Москва, Россия.

1) Работа поддерживается РФФИ, грант 96-01-01129 и внебюджетным фондом НИР МПС РФ. 
Средние Рисса поля $X(t)$ со сферически симметричным весом $b_{1}(|t|)$ определяются формулой

$$
S_{r}=B_{1}^{-1}(r) \int_{|t| \leqslant r} b_{1}(|t|) X(t) d t .
$$

В частности, при $b_{1}(t)=1$ это средние арифметические поля

$$
\Sigma_{r}=V_{k}^{-1} r^{-k} \int_{|t| \leqslant r} X(t) d t .
$$

В работе [1] показано, что средние $\Sigma_{\tau}$ имеют -представление

$$
\Sigma_{r}=Z\left[|\lambda| \leqslant a_{m}\right]+\eta_{r} \quad \text { при } \quad 2^{m} \leqslant r<2^{m+1},
$$

где

$$
a_{m}=2^{-m} \quad \text { и } \quad \lim _{r \rightarrow \infty} \dot{\eta}_{r}=0 \quad \text { п.в., }
$$

а $Z[A]=\int_{A} Z(d \lambda)$ для борелевских множеств $A$ в $\mathbf{R}^{k}$.

В частности, отсюда следует, что не все однородные случайные поля суммируются почти всюду методом средних арифметических, и для их суммирования в ряде случаев нужны более сильные методы.

В случае $k=1$ для стапионарных процессов с дискретным временем в работе [2] изучалась сходимость почти всюду средних Рисса $B_{N}^{-1} \sum_{k=1}^{N} b_{k} X_{k}$, где $B_{N}=\sum_{k=1}^{N} b_{k} \longrightarrow \infty$ при $N \rightarrow \infty$. При этом на коэффициенты $b_{k}$ налагались ограничения типа $b_{k}=g(k) k^{-1}$, где $g(k)-$ медленно меняющаяся функция.

Цель данной работы - обобщить этот результат на однородные случайные поля любой размерности и расширить множество допустимых весовых функций (в частности, при $k=1$ рассматриваются средние Рисса с любыми невозрастающими весами).

Сделаем сначала несколько замечаний о взаимосвязи $B$-средних со средними арифметическими. Пусть $Y_{1}(t)$ - любая локально интегрируемая функция из $\mathbf{R}^{k}$ в $\mathbf{R}, t=\left(t_{1}, \ldots, t_{k}\right)$. Пусть функции $b_{1}(u), B_{1}(u)$, $b(u), B(u)$ те же, что и прежде. $B$-средние функции $Y_{1}(t)$ и ее средние арифметические имеют вид

$$
\begin{aligned}
& S(r)=B_{1}^{-1}(r) \int_{|t| \leqslant r} b_{1}(|t|) Y_{1}(t) d t, \\
& \Sigma(r)=V_{k}^{-1} r^{-k} \int_{|t| \leqslant r} Y_{1}(t) d t .
\end{aligned}
$$

Переходя к сферическим координатам и, обозначая якобиан преобразования $c_{k} \rho^{k-1} I\left(\varphi_{1}, \ldots, \varphi_{k-1}\right)$ и полагая

$$
\int Y_{1}\left(\rho, \varphi_{1}, \ldots, \varphi_{k-1}\right) I\left(\varphi_{1}, \ldots, \varphi_{k-1}\right) d \varphi_{1} \ldots d \varphi_{k-1}=Y(\rho)
$$


получим

$$
\begin{aligned}
& S(r)=B^{-1}(r) \int_{0}^{r} b(\rho) Y(\rho) d \rho \\
& \Sigma(r)=k r^{-k} \int_{0}^{r} \rho^{k-1} Y(\rho) d \rho
\end{aligned}
$$

где функция $b(u)$ задается формулой $(2)$.

Справедлива следуюшая просгая и, вероятно, не новая лемма.

Лемма 1. Пусть для дифференцируемой положительной функции $b_{1}(u)$ выполнены условия: $\left(\exists u_{0}>0\right)$

$$
\begin{aligned}
& (\exists \alpha \geqslant 0): \quad u^{-\alpha} b_{1}(u) \quad \text { не возрастает пр } u \quad u \geqslant u_{0}, \\
& (\exists 0<\beta<k): \quad u^{\beta} b_{1}(u) \quad \text { не убывает пр } u \quad u \geqslant u_{0} .
\end{aligned}
$$

Тогда B-метод эквивалентеи методу $(C, 1)$, т.е. для любой локально интегрируемой функци $Y(t)$ эквивалентны условия:

$$
s(r)=\int_{0}^{r} t^{k-1} b_{1}(t) Y(t) d t=o(B(r)) \quad n p u \quad r \rightarrow \infty
$$

$u$

$$
\sigma(r)=\int_{0}^{r} t^{k-1} Y(t) d t=o\left(r^{k}\right) \quad \text { npu } \quad r \rightarrow \infty .
$$

Д ок а з а т л ь с т о. Из (7) и (8) сразу следует, что

$$
c_{1} u^{k} b_{1}(u) \leqslant B(u)=\int_{0}^{u} t^{k-1} b_{1}(t) d t \leqslant c_{2} u^{k} b_{1}(u)
$$

Условия $\left(u^{-\alpha} b_{1}(u)\right)^{\prime} \leqslant 0$ и $\left(u^{\beta} b_{1}(u)\right)^{\prime} \geqslant 0$ влекут за собой, что при $u \geqslant u_{0}$

$$
\left|u b_{1}^{\prime}(u)\right| \leqslant C_{3} b_{1}(u), \quad C_{3}=\max (\alpha, \beta) .
$$

Іокажем, что из (9) можно вывести (10). Из (9) и (12) находим, что

$$
\begin{aligned}
\sigma(r) & =\int_{0}^{r} b_{1}^{-1}(t) d s(t)=s(r) b_{1}^{-1}(r)+\int_{0}^{r} b_{1}^{-2}(t) b_{1}^{\prime}(t) s(t) d t \\
& =o\left(b_{1}^{-1}(r) B(r)\right)+o\left(\int_{u_{0}}^{r} b_{1}^{-1}(t) t^{-1} B(t) d t\right)
\end{aligned}
$$

Используя (11), заключаем, что оба слагаемых в правой части последнего равенства имеют порядок $o\left(r^{k}\right)$ при $r \rightarrow \infty$. Аналогично доказывается, что (10) влечет за собой (9).

Из леммы 1 следует, что если интересоваться $B$-методами более сильными, чем метод $(C, 1)$, то функция $b_{1}(u)$ должна иметь вид

$$
b_{1}(u)=\varphi(u) u^{-k}
$$

где $\varphi(u)$ растет не быстрее $u^{\varepsilon}$ при любом $\varepsilon>0$. 
Пусть функция $b(u)$ определена формулой (2). Далее предполагается, что выполнено условие:

$$
b(u) \text { не возрастает при } u \geqslant u_{0} .
$$

В частности, в этот класс входят все функции вида (13), где $\varphi(u)-$ медленно меняюшаяся функция.

Подстазим спектральное разложение (1) поля $X(t)$ в формулу (4). После интегрирования по угловым координатам средние $S_{r}$ представляются в виде

$$
S_{r}=\int_{\mathbf{R}^{k}} Q_{r}(\lambda) Z(d \lambda)
$$

где

$$
Q_{r}(\lambda)=(B(r))^{-1} \int_{0}^{r} b(u) D(u|\lambda|) d u
$$

ядро $D(v)$ выражается через бесселеву функцию $J_{\nu}(v), \nu=k / 2-1$, в виде

$$
D(v)=a_{\nu} J_{\nu}(v) v^{-\nu}, \quad a_{\nu}=2^{\nu} \Gamma(\nu+1) .
$$

Далее через $C$ будут обозначаться постоянные, зависяшие от $k$, не обязательно одинаковые в разных неравенствах.

Лемма 2. Если выполнено условие (14), то для $x>0, N_{0}<N<M$

$$
\left|\int_{N}^{M} b(u) D(u x) d u\right| \leqslant C b(N) x^{-1}
$$

Док аз ат ельст в о. При $k=1$

$$
D(u)=u^{1 / 2} J_{1 / 2}(u)=\left(2 \pi^{-1}\right)^{1 / 2} \cos u .
$$

При $k \geqslant 2$ известна асимптотика

$$
D(u)=h_{\nu} u^{-(\nu+1 / 2)} \cos \left(u+b_{\nu}\right)+o\left(u^{-(\nu+3 / 2)}\right), \quad \nu=\frac{k}{2}-1 .
$$

Поэтому

$$
|\Phi(v)|=\left|\int_{0}^{v} D(u) d u\right| \leqslant C \quad \text { при } \quad v>0 .
$$

Из условий (14) и (19) вытекает (18).

Лемма 3. При любых $x>0$ u $N>0$ справедлива оченка

$$
\left|\int_{0}^{N} b(u) D(u x) d u\right| \leqslant C\left(B\left(x^{-1}\right)+1\right) .
$$

Д ок а $а$ а т е л ь с т в о. Представим интеграл в левой части в виде суммы интегралов по промежуткам $\left(0, u_{0}\right),\left(u_{0}, x^{-1}\right),\left(x^{-1}, N\right)$; некоторые слагаемые могут отсутствовать, например, при $N \leqslant x^{-1}$. 
Так как $|D(u)| \leqslant C$, то

$$
\begin{aligned}
& \left|\int_{0}^{u_{0}} b(u) D(u x) d u\right| \leqslant C \\
& \left|\int_{u_{0}}^{x^{-1}} b(u) D(u x) d u\right| \leqslant C \int_{0}^{x^{-1}} b(u) d u=C B\left(x^{-1}\right) .
\end{aligned}
$$

Далеe,

$$
B(v)=\int_{0}^{v} b(u) d u \geqslant\left(v-u_{0}\right) b(v)
$$

и по лемме 2 при $N>x^{-1}$

$$
\left|\int_{x^{-1}}^{N} b(u) D(u x) d u\right| \leqslant C b\left(x^{-1}\right) x^{-1} \leqslant C\left[B\left(x^{-1}\right)+1\right] .
$$

Объединив эти оценки, получим неравенство (20).

Введем следующие обозначения. Так как функция $B(r)$ монотонно возрастает от 0 до $+\infty$, для $m=1,2, \ldots$ обозначим через $N_{m}^{\prime}$ корень уравнения $B(u)=m$; положим также $N_{m}=N_{2^{m}}^{\prime}$, т.е. $B\left(N_{m}\right)=2^{m}$; пусть

$$
\lambda_{m}=N_{m+1}^{-1} \quad \text { при } \quad m \geqslant 0, \quad N_{2^{m}+n}^{\prime}=N_{m, n}, \quad 0 \leqslant n<2^{m} .
$$

Заметим, что из условия (14) вытекает, что $N_{m+1} \geqslant 2 N_{m}-C$, и поэтому

$$
\sum_{k=1}^{m} N_{k}^{\alpha} \leqslant C N_{m}^{\alpha}, \quad \sum_{k=m+1}^{\infty} N_{k}^{-\alpha} \leqslant C N_{m}^{-\alpha} \quad(\forall \alpha>0) .
$$

Теорема 1. Если весовая функция $b_{1}(u)$ удовлетворяет соотношению (14), то средние Рисса любого однородного случайного поля допускают следующее представление при $N_{m} \leqslant r<N_{m+1}$ :

$$
S_{r}=Z\left[|\lambda| \leqslant \lambda_{m}\right]+\eta_{r}, \quad \lim _{r \rightarrow \infty} \eta_{r}=0 \quad \text { n.в. }
$$

Доказательст в о. Положим

$$
\begin{aligned}
& \Delta_{m}^{\prime}=\sup \left\{m^{-1}\left|\int_{N_{m}^{\prime} \leqslant|t| \leqslant r} b_{1}(|t|) X(t) d t\right| ; N_{m}^{\prime} \leqslant r<N_{m+1}^{\prime}\right\}, \\
& \Delta_{m}=\sup \left\{\left|S_{N_{m, n}}-Z\left[|\lambda| \leqslant \lambda_{m}\right]\right| ; 0 \leqslant n<2^{m}\right\} .
\end{aligned}
$$

Для доказательства теоремы достаточно проверить равенства

$$
\begin{array}{ll}
\lim _{m \rightarrow \infty} \Delta_{m}^{\prime}=0 & \text { п.в., } \\
\lim _{m \rightarrow \infty} \Delta_{m}=0 & \text { п.в. }
\end{array}
$$


Из неравенства Коши-Буняковского и определения чисел $N_{m}^{\prime}$ следует оценка

$$
\left\|\Delta_{m}^{\prime}\right\|^{2} \leqslant m^{-2}\left\{\left\|\int_{N_{m}^{\prime} \leqslant|t|<N_{m+1}^{\prime}} b_{1}(|t|)|X(t)| d t\right\|^{2}\right\} \leqslant C \sigma^{2} m^{-2} .
$$

Поэтому $\sum\left\|\Delta_{m}^{\prime}\right\|^{2}<\infty$ и выполнено (23). Опенка выражения $\Delta_{m}$ проводится по той же схеме «двоичного разложения сумм», что и в работе [2], и опирается на полученные выше свойства ядер $D(u)$. Положим

$$
\begin{gathered}
b(u)(D(u|\lambda|)-1)=p(u, \lambda), \quad b(u) D(u|\lambda|)=q(u, \lambda), \\
N_{m p j}=N_{2^{m}+j 2^{m-p}}^{\prime}, \quad j=0, \ldots, 2^{p}, \quad p=1,2, \ldots, m .
\end{gathered}
$$

Из (15), (16) следует, что

$$
\left|S_{N_{m, n}}-Z\left[|\lambda| \leqslant \lambda_{m}\right]\right|=\left|J_{m, n}^{1}\right|+\left|J_{m, n}^{2}\right|,
$$

где

$$
\begin{aligned}
& J_{m, n}^{1}=2^{-m} \int_{|\lambda| \leqslant \lambda_{m}}\left(\int_{0}^{N_{m, n}} p(u, \lambda) d u\right) Z(d \lambda), \\
& J_{m, n}^{2}=2^{-m} \int_{|\lambda|>\lambda_{m}}\left(\int_{0}^{N_{m, n}} q(u, \lambda) d u\right) Z(d \lambda) .
\end{aligned}
$$

Положим также

$$
\begin{aligned}
f_{m p j}(\lambda) & =\int_{N_{m p(j-1)} \leqslant|u|<N_{m p j}} p(u, \lambda) d u \\
g_{m p j}(\lambda) & =\int_{N_{m p(j-1)} \leqslant|u|<N_{m p j}} q(u, \lambda) d u \\
\Delta_{m p j}^{1} & =\int_{|\lambda| \leqslant \lambda_{m}} f_{m p j}(\lambda) Z(d \lambda), \quad \Delta_{m p j}^{2}=\int_{|\lambda|>\lambda_{m}} g_{m p j}(\lambda) Z(d \lambda), \\
\varepsilon_{m}^{1} & =\int_{|\lambda| \leqslant \lambda_{m}}\left(\int_{0}^{N_{m}} p(u, \lambda) d u\right) Z(d \lambda), \\
\varepsilon_{m}^{2} & =\int_{|\lambda|>\lambda_{m}}\left(\int_{0}^{N_{m}} q(u, \lambda) d u\right) Z(d \lambda) .
\end{aligned}
$$

Для проверки свойства (24) достаточно, как и в работе [2], доказать, что сходятся четыре ряда

$$
\Sigma_{i}=\sum 2^{-2 m}\left\|\varepsilon_{m}^{i}\right\|^{2} \quad \text { и } \quad \Sigma_{i}^{\prime}=\sum 2^{-2 m} \gamma_{m}^{i}, \quad i=1,2,
$$

где

$$
\gamma_{m}^{i}=\sum_{p=1}^{m} p^{2} \sum_{j=1}^{2^{p}}\left\|\Delta_{m p j}^{i}\right\|^{2}, \quad i=1,2
$$


Кроме лемм 1, 2 при оценке используются следуюшие очевидные неравенства:

$$
\begin{gathered}
|D(u)| \leqslant C, \\
|D(u)-1| \leqslant C u^{2}
\end{gathered}
$$

Пусть $F(d \lambda)=\|Z(d \lambda)\|^{2}, \int_{\mathbf{R}^{k}} F(d \lambda)<\infty$, и будем пользоваться известным свойством спектральной меры

$$
\left\|\int g(\lambda) Z(d \lambda)\right\|^{2}=\int|g(\lambda)|^{2} F(d \lambda) \quad \text { для } \quad g \in L_{F}^{2} .
$$

О ц е н к а р я д а $\Sigma_{1}$. В силу $(26)$ при $N_{m}|\lambda| \leqslant 1$ имеем:

$$
\left|\int_{0}^{N_{m}} p(u, \lambda) d u\right| \leqslant C N_{m}^{2}|\lambda|^{2} \int_{0}^{N_{m}} b(u) d u \leqslant C N_{m}|\lambda| 2^{m}
$$

Далее часто употребляются следующие обозначения:

$$
A_{0}=\int_{|\lambda|>\lambda_{1}} F(d \lambda), \quad A_{k}=\int_{\lambda_{k+1}<|\lambda| \leqslant \lambda_{k}} F(d \lambda), \quad \sum A_{k}<\infty .
$$

Так как $\lambda_{k}=N_{k+1}^{-1}$, то из (27) следует, что

$$
\begin{aligned}
\sum_{m=1}^{\infty} 2^{-2 m}\left\|\varepsilon_{m}^{1}\right\|^{2} & \leqslant C \sum_{m=1}^{\infty} N_{m}^{2} \sum_{k=m}^{\infty} \int_{\lambda_{k+1}<|\lambda| \leqslant \lambda_{k}}|\lambda|^{2} F(d \lambda) \\
& \leqslant C \sum_{m=1}^{\infty} N_{m}^{2} \sum_{k=m}^{\infty} N_{k+1}^{-2} A_{k} \leqslant \sum_{k=1}^{\infty} A_{k} N_{k+1}^{-2} \sum_{m=1}^{k} N_{m}^{2}
\end{aligned}
$$

и ряд $\Sigma_{1}$ сходится в силу (21) и (28).

О ц е н к а р я д а $\Sigma_{2}$. Положим $Q_{m}(\lambda)=\int_{0}^{N_{m}} q(u, \lambda) d u$. Тогда

$$
\left\|\varepsilon_{m}^{2}\right\|^{2}=\int_{\lambda_{m}<|\lambda| \leqslant \lambda_{1}}\left|Q_{m}(\lambda)\right|^{2} F(d \lambda)+\int_{|\lambda|>\lambda_{1}}\left|Q_{m}(\lambda)\right|^{2} F(d \lambda) .
$$

Из леммы 3 следуют оценки:

$$
\left|Q_{m}(\lambda)\right| \leqslant C B\left(|\lambda|^{-1}\right) \quad \text { при } \quad|\lambda| \leqslant \lambda_{1}, \quad\left|Q_{m}(\lambda)\right| \leqslant C \quad \text { при } \quad|\lambda|>\lambda_{1} .
$$

Подставляя эти ощенки в (29), имеем:

$$
\sum_{m=1}^{\infty} 2^{-2 m}\left\|\varepsilon_{m}^{2}\right\|^{2} \leqslant C+C \sum_{m=2}^{\infty} 2^{-2 m} \sum_{k=1}^{m-1} \int_{\lambda_{k+1}<|\lambda| \leqslant \lambda_{k}} B^{-2}\left(|\lambda|^{-1}\right) F(d \lambda)
$$


Но на интервале $\left(\lambda_{k+1}, \lambda_{k}\right)$

$$
B\left(|\lambda|^{-1}\right) \leqslant B\left(\lambda_{k+1}^{-1}\right)=B\left(N_{k+2}\right)=2^{k+2} .
$$

Поэтому ряд в правой части неравенства (30) мажорируется рядом

$$
C \sum_{m=2}^{\infty} 2^{-2 m} \sum_{k=1}^{m-1^{-}} A_{k} 2^{2 k} \leqslant C \sum_{k=1}^{\infty} A_{k}<\infty .
$$

О ц е н к а ря д а $\Sigma_{1}^{\prime}$. Поо определению чисел $N_{m p j}$ и в силу (26) при $|\lambda| \leqslant \lambda_{m}$ получаем

$$
\left|f_{m p j}(\lambda)\right| \leqslant C N_{m p j}^{2}|\lambda|^{2} \int_{N_{m p(j-1)}}^{N_{m p j}} b(u) d u \leqslant C N_{m+1}|\lambda| 2^{m-p} .
$$

Поэтому

$$
\left\|\Delta_{m p j}^{1}\right\|^{2} \leqslant C N_{m+1}^{2} 2^{2 m-2 p} \int_{|\lambda| \leqslant \lambda_{m}}|\lambda|^{2} F(d \lambda),
$$

и ряд $\Sigma_{1}^{\prime}$ мажорируется рядом

$$
C \sum_{m=1}^{\infty} \sum_{p=1}^{m} p^{2} 2^{-p} N_{m+1}^{2} \sum_{k=m+1}^{\infty} A_{k} N_{k}^{-2} \leqslant C \sum_{k=1}^{\infty} A_{k}<\infty .
$$

О ц е н к а ря д а $\Sigma_{2}^{\prime}$. Положим

$$
\begin{aligned}
\left\|\Delta_{m p j}^{2}\right\|^{2} & =\alpha_{m p j}+\beta_{m p j}, \\
\alpha_{m p j} & =\int_{\lambda_{m}<|\lambda| \leqslant \lambda_{m-p}}\left|g_{m p j}(\lambda)\right|^{2} F(d \lambda), \\
\beta_{m p j} & =\int_{|\lambda|>\lambda_{m-p}}\left|g_{m p j}(\lambda)\right|^{2} F(d \lambda), \\
\alpha_{m} & =2^{-2 m} \sum_{p=1}^{m} p^{2} \sum_{j=1}^{2^{p}} \alpha_{m p j}, \quad \beta_{m}=2^{-2 m} \sum_{p=1}^{m} p^{2} \sum_{j=1}^{2^{p}} \beta_{m p j} .
\end{aligned}
$$

Надо проверить, что $\sum \alpha_{m}<\infty$ и $\sum \beta_{m}<\infty$. Из (25) следует, что

$$
\begin{aligned}
\left|g_{m p j}(\lambda)\right| & =\left|\int_{N_{m p(j-1)}}^{N_{m p j}} b(u) D(u|\lambda|) d u\right| \leqslant C 2^{m-p}, \\
\alpha_{m p j} & \leqslant C 2^{2 m-2 p} \sum_{k=m-p}^{m-1} A_{k}, \quad \sum \alpha_{m} \leqslant C \sum_{m=1}^{\infty} \sum_{p=1}^{m} p^{2} 2^{-p} \sum_{k=m-p}^{m-1} A_{k} \\
& \leqslant C \sum_{k=0}^{\infty} A_{k} \sum_{m=k+1}^{\infty}(m-k)^{2} 2^{-(m-k)}<\infty .
\end{aligned}
$$


При оценке $\beta_{m}$ используем лемму 2 и свойство (14). При $1 \leqslant j \leqslant$ $2^{p}, \quad p \leqslant m$

$$
\left|g_{m p j}(\lambda)\right| \leqslant C|\lambda|^{-1} b\left(N_{m p(j-1)}\right) \leqslant C b\left(N_{m}\right)|\lambda|^{-1}
$$

Поэтому

$$
\beta_{m p j} \leqslant C b^{2}\left(N_{m}\right) \int_{|\lambda|>\lambda_{m-p}}|\lambda|^{-2} F(d \lambda) \leqslant C b^{2}\left(N_{m}\right) \sum_{k=0}^{m-p-1} A_{k} N_{k+2}^{2}
$$

Используя (14), получаем:

$$
\begin{aligned}
\sum_{m=2}^{\infty} \beta_{m} & \leqslant C \sum_{m=2}^{\infty} b^{2}\left(N_{m}\right) 2^{-2 m} \sum_{p=1}^{m} p^{2} 2^{p} \sum_{k=0}^{m-p-1} A_{k} N_{k+2}^{2} \\
& =C \sum_{m=2}^{\infty} b^{2}\left(N_{m}\right) 2^{-2 m} \sum_{k=0}^{m-2} A_{k} N_{k+2}^{2}(m-k)^{2} 2^{m-k} \\
& \leqslant C \sum_{k=0}^{\infty} A_{k} N_{k+2}^{2} 2^{-2 k} \sum_{m=k+2}^{\infty} b^{2}\left(N_{m}\right)(m-k)^{2} 2^{-(m-k)} \\
& \leqslant C \sum_{k=0}^{\infty} A_{k} N_{k+2}^{2} b^{2}\left(N_{k+2}\right) 2^{-2 k} \leqslant C \Sigma A_{k}<\infty
\end{aligned}
$$

так каK

$$
N_{k+2} b\left(N_{k+2}\right) \leqslant C B\left(N_{k+2}\right) \leqslant C 2^{k} \text {. }
$$

Итак, ряды $\Sigma_{i}$ и $\Sigma_{i}^{\prime}(i=1,2)$ сходятся, и оценка $(24)$ доказана. Из (23) и (24) вытекает нужное представление (22) средних $S_{r}$.

Следствие 1. Если $\left(X(t), t \in \mathbf{R}^{1}\right)$ - стачионарный прочесс, то для любых невозрастающих весов $b_{1}(t)$ для В-средних Рисса справедливо представление (22).

При $b_{1}(t)=1$ следствие 1 дает представление средних арифметических $(5),(6)$; для процессов с дискретным временем и весов $b_{1}(t)=$ $t^{-1} g(t)$, где $g(t)$ - медленно меняющаяся функция, этот результат получен в работе [2].

Теорема 2. Если для однородного поля $X(t)$ выполнено условие

$$
\int_{|\lambda|>0}\left(\ln \ln B\left(|\lambda|^{-1}\right)\right)^{2} F(d \lambda)<\infty, \quad F(\{0\})=0
$$

mо B-средние поля сходятся n.в., $\lim _{r \rightarrow \infty} S_{r}=0$ n.в.

Условие (31) нельзя ослабить: если $w(u)$ - неубывающая функция, $w(u)=o\left(\ln ^{2} \ln u\right)$, по существует однородное поле, для которого

$$
\int_{|\lambda|>0} w\left(B\left(|\lambda|^{-1}\right)\right) F(d \lambda)<\infty, \quad \text { но } \limsup _{r \rightarrow \infty}\left|S_{r}\right|=\infty \quad \text { n.в. }
$$


Это следствие из теоремы 1 выводится, как и в работах [1], [2], из теоремы Меньшова-Радемахера о сходимости п.в. ортогонального ряда $\sum \Phi_{k}, \Phi_{k}=Z\left[\lambda_{k+1}<|\lambda| \leqslant \lambda_{k}\right]$, при условии

$$
\sum\left\|\Phi_{k}\right\|^{2} \ln ^{2} k<\infty
$$

Достаточное условие сходимости средних $S_{r}$ можно выразить также через корреляционную функцию поля $K(u)$. Удобно ввести усредненную корреляционную функцию $K_{1}(u)$ :

и

$$
K_{1}(\rho)=\left|V_{\rho}\right|^{-1} \int_{|u| \leqslant \rho} K(u) d u
$$

$$
K_{2}(\rho)=\left|V_{\rho}\right|^{-2} \mathbf{E}\left|\int_{|u| \leqslant \rho} X(u) d u\right|^{2},
$$

где $V(\rho)$ объем шара радиуса $\rho$ в $\mathbf{R}^{k}$. Пусть $B^{*}(\rho)=\ln ^{2} \ln B(\rho)$, когда $B(\rho) \geqslant e, B^{*}(\rho)=0$ при $B(\rho) \leqslant e$.

Теорема 3. Eсли при $i=1$ или $i=2$

$$
\int_{e}^{\infty} K_{i}(\rho) d B^{*}(\rho)<\infty,
$$

то средние Pисcа $S_{r}$ сxодяmcя n.6. при $r \rightarrow \infty$.

Д о к а з а те ль с т во. Так как

To

$$
K(u)=\int_{\mathbf{R}^{k}} e^{i u \lambda} F(d \lambda)
$$

$$
K_{1}(\rho)=\int_{\mathbf{R}^{k}} Q(\rho|\lambda|) F(d \lambda),
$$

где $Q(u)=b_{k} u^{-k / 2} J_{k / 2}(u)$.

Из свойств ядра $Q(u)$ легко следует, что

$$
\begin{aligned}
& G(\rho)=\int_{|\lambda| \leqslant \rho^{-1}} Q(\rho|\lambda|) F(d \lambda) \geqslant C_{1} \int_{|\lambda| \leqslant \rho^{-1}} F(d \lambda), \\
& H(\rho)=\int_{|\lambda| \geqslant \rho^{-1}} Q(\rho|\lambda|) F(d \lambda) \leqslant C_{2} \int_{|\lambda| \geqslant \rho^{-1}}|\lambda|^{-1} \rho^{-1} F(d \lambda) .
\end{aligned}
$$

В силу (14) имеем:

$$
\left[B^{*}(\rho)\right]^{\prime}=2[B(\rho) \ln B(\rho)]^{-1} \ln \ln B(\rho) b(\rho) \leqslant C_{3} \rho^{-1}, \quad \rho \geqslant \rho_{0} .
$$


Из (34), (35) получаем, что

$$
\begin{aligned}
\int_{\rho_{0}}^{\infty} H(\rho) d B^{*}(\rho) & \leqslant C \int_{\rho_{0}}^{\infty} \rho^{-2} \int_{|\lambda| \geqslant \rho^{-1}}|\lambda|^{-1} F(d \lambda) d \rho \\
& \leqslant C \int_{\mathbf{R}^{k}} F(d \lambda)<\infty
\end{aligned}
$$

Так как $\left|K_{1}(\rho)-G(\rho)\right| \leqslant H(\rho)$, то из (36) и условия (32) при $i=1$ вытекает, что

$$
\int_{\rho_{0}}^{\infty} G(\rho) d B^{*}(\rho)<\infty
$$

Имеем:

$$
\begin{aligned}
\int_{|\lambda| \leqslant e^{-1}} B^{*}\left(|\lambda|^{-1}\right) F(d \lambda) & =\int_{|\lambda| \leqslant e^{-1}}\left(\int_{0}^{|\lambda|^{-1}} d B^{*}(\rho)\right) F(d \lambda) \\
& =\int_{e}^{\infty}\left(\int_{|\lambda| \leqslant \rho^{-1}} F(d \lambda)\right) d B^{*}(\rho) .
\end{aligned}
$$

В силу (33) и (37) последний интеграл конечен, и можно применить теорему 2. С другой стороны, легко проверяется оценка

$$
K_{2}(\rho) \geqslant \int_{|\lambda| \leqslant \rho^{-1}} F(d \lambda)
$$

и из условия (32) при $i=2$ также получаем (31).

Следствие 2. Если $K(u)-$ коррелячионная функция случайного поля $X(t)$ и выполнены соотношения

$$
\begin{aligned}
|K(u)| & \leqslant \Phi(|u|) \\
\int_{\rho_{0}}^{\infty} \Phi(\rho) d B^{*}(\rho) & <\infty
\end{aligned}
$$

и при некотором $\alpha<k$ бункчия $u^{\alpha} \Phi(u)$ не убывает, mо $\lim _{r \rightarrow \infty} S_{r}$ суиествует почти встоду.

Действительно, из этих условий вытекает, что

$$
\left|K_{1}(\rho)\right| \leqslant C \rho^{-k} \int_{0}^{\rho} u^{k-1} \Phi(u) d u \leqslant C \Phi(\rho) \quad\left(\rho \geqslant \rho_{0}\right) .
$$

Таким образом, условие (39) влечет за собой условие (32) при $i=1$. Для важного частного случая - логарифмических средних Рисса из теорем 2 и 3 получаем такой результат.

Обозначим логарифмические средние поля $X(t)$ через $S_{r}^{*}$,

$$
S_{r}^{*}=C_{k}(\ln r)^{-1} \int_{1 \leqslant|t| \leqslant r}|t|^{-k} X(t) d t
$$


Следствие 3. Eсли при некотором $\lambda_{0}>0$ выполнено условие

$$
\int_{0<|\lambda| \leqslant \lambda_{0}}\left(\ln \ln \ln |\lambda|^{-1}\right)^{2} F(d \lambda)<\infty, \quad F(\{0\})=0
$$

или при некотором $\varepsilon>0$ выполнено условие

$$
|K(u)|=o(\ln \ln \ln |u|)^{-(2+\varepsilon)} \quad n p u \quad u \rightarrow \infty
$$

mо $\lim _{r \rightarrow \infty} S_{r}^{*}=0$ п.в. При этом в условии (40) мельзя заменить подынтегральную функчию на функцию $w(u)=o(\ln \ln \ln u)^{2}$, а в условии (41) нельзя положить $\varepsilon=0$.

Теорема 4. Для любого однородного поля существует метод Рисса, суммирующий это поле почти всюду.

Так как $\int_{\mathbf{R}^{k}} F(d \lambda)<\infty$, то существует достаточно медленно стремящаяся к $\infty$ функция $B(u)$, произвоцная которой $b(u)$ не возрастает, и интеграл $\int_{\mathbf{R}^{k}} \ln ^{2} \ln B\left(|\lambda|^{-1}\right) F(d \lambda)$ сходится. Положим $b_{1}(u)=b(u) u^{-k+1}$ и образуем $B$-средние поля по формуле (4). По теореме 2 они сходятся Ir.B.

Как и в работах [1]--[5], можно показать, что результаты данной статьи переносятся на полугруппы унитарных или нормальных операторов (зависящих от одного или нескольких параметров) или на гармонизуемые в том или ином смысле случайные поля.

\section{СПИСОК ЛИТЕРАТУРЫ}

1. Гапошкин $B$. $Ф$. Критерии усиленного закона больших чисел для классов стащионарных в широком смысле процессов и однородных случайных полей. - Теория вероятн. и ее примен., 1977, т. 22, в. 2, с. 295-319.

2. Гапошкин $B . \Phi$. О суммировании стационарных последовательностей методами Рисса. - Матем. заметки, 1995, т. 32, № 1, с. 653-662.

3. Гапошкин $B . \Phi$. Об индивидуальной эргодической теореме для нормальных операторов. - Функц. анализ и его прилож., 1981, т. 15, № 2, с. 131-142.

4. Houdré $C$. Harmonizability, $V$-boundedness, $(2, p)$-boundedness of stochastic processes. - Probab. Theory Relat. Fields, 1990, v. 84, p. 39-45.

5. Berkson E., Bourgain J., Gillespie T. A. On the a.e. convergence of ergodic averages for power-bounded operators on $L^{p}$-spaces. - Int. Eq. Operat. Th., 1991, v. 14, p. 678-715. 